Original Paper http://ajol.info/index.php/ijbcs $\quad$ http://indexmedicus.afro.who.int

\title{
Activité analgésique de quatre plantes utilisées dans la prise en charge traditionnelle du paludisme au Mali et au Togo
}

\author{
Adama DENOU ${ }^{1 *}$, Koffi KOUDOUVO ${ }^{2}$, Mahamane HAIDARA $^{1}$, Adiaratou TOGOLA ${ }^{2}$, \\ Rokia SANOGO $^{1,2}$, Komlan ESSIEN ${ }^{3}$, Kodjo A. AKLIKOKOU ${ }^{3}$, Drissa DIALLO ${ }^{1,2}$ et \\ Messanvi GBEASSOR ${ }^{3,4}$ \\ ${ }^{1}$ Faculté de Pharmacie, Université des Sciences, des Techniques et des Technologies de Bamako, \\ BP:1805, Bamako, Mali. \\ ${ }^{2}$ Département de Médecine Traditionnelle (DMT), Institut National de Recherche en Santé Publique (INRSP), \\ BP:1746, Bamako, Mali. \\ ${ }^{3}$ Laboratoire de Physiologie Animale et de Pharmacologie des Sciences Naturelles, \\ Faculté des Sciences, Université de Lomé, BP: 1515, Lomé, Togo. \\ ${ }^{3}$ Centre de Recherche et de Formation sur les Plantes Médicinales (CERFOPLAM), \\ Université de Lomé, BP:1515, Lomé, Togo. \\ *Auteur correspondant; E-mail: adamadenou@yahoo.fr; DMT B.P.1746 Bamako. Tel. : (00223)76309543
}

\section{RÉSUMÉ}

En Afrique, plusieurs personnes utilisent les plantes médicinales pour traiter l'inflammation et la douleur. L'objectif du présent travail était d'évaluer l'effet analgésique de Cymbopogon citratus (DC) Stapf., Cleome viscosa Linn., Mitragyna inermis (Willd.) O. Kuntze et Sclerocarya birrea (A. rich.) Hochst utilisées dans la prise en charge traditionnelle du paludisme au Mali et au Togo. Le matériel végétal (feuilles, rameau feuillé, écorce de tronc et partie aérienne) des espèces a été récolté au Mali et au Togo. Les extraits aqueux du matériel végétal ont été préparés pour réaliser d'une part l'analyse phytochimique à l'aide de la chromatographie sur couche mince et d'autre part l'évaluation de leur activité analgésique sur la douleur induite avec l'acide acétique chez les souris. L'analyse phytochimique a révélé que les extraits de plantes contenaient des tanins, flavonoïdes, alcaloïdes, stérols et terpènes, et des saponosides. Dans l'ensemble, toutes les plantes protègent contre la douleur. Cependant, Cymbopogon citratus et Mitragyna inermis ont présenté les meilleures protections. Les propriétés analgésiques des extraits aqueux de ces plantes ont un grand intérêt dans la prise en charge traditionnelle du paludisme.

(c) 2016 International Formulae Group. All rights reserved.

Mots clés: Plantes antipaludiques, propriétés analgésiques, Mali, Togo.

\section{Analgesic activity of four plants used in traditional management of malaria in Mali and in Togo}

\section{ABSTRACT}

In Africa, many people use medicinal plants to treat inflammation and pain. The objective of the present work was to evaluate the analgesic effect of Cymbopogon citratus (DC) Stapf, Cleome viscosa Linn., Mitragyna inermis (Willd.) O. Kuntze and Sclerocarya birrea (A. rich.) Höchst used in traditional care of 
malaria in Mali and Togo. The plant material (leaves, leaved twig, stem bark and aerial part) species has been collected in Mali and Togo. The aqueous extracts of plant material were prepared to achieve firstly the phytochemical analysis using thin layer chromatography (TLC) and on the other hand the assessment of their analgesic activity on pain induced with acetic acid in mice. Phytochemical analysis revealed that plant extracts contain tannins, flavonoids, alkaloids, sterols and terpens, and saponins. All plants protect against pain. However Cymbopogon citratus and Mitragyna inermis presented the best protections. The analgesic properties of aqueous extracts of these plants have a great interest in the traditional management of malaria.

(C) 2016 International Formulae Group. All rights reserved.

Keywords: Analgesic properties, antimalarial plants, Mali, Togo.

\section{INTRODUCTION}

En médecine traditionnelle, de nombreuses recettes sont utilisées dans la prise charge symptomatique du paludisme. C'est ainsi qu'une grande majorité de plantes utilisées dans le traitement traditionnel du paludisme sont indiquées dans la prise en charge de la fièvre et la douleur.

Des enquêtes ethnobotaniques nous ont permis de recenser au Mali et au Togo, 126 plantes utilisées dans la prise en charge traditionnelle de la douleur palustre. Selon la méthode de sélection progressive propre à l'Achat en Triplet de Plantes Médicinales (ATRM), Cymbopogon citratus et Cleome viscosa, utilisées au Togo; Mitragyna inermis et Sclerocarya birrea, employées au Mali, ont été choisies pour vérifier leurs propriétés analgésiques sur des modèles de laboratoires.

De nombreuses études pharmacologiques et phytochimiques ont déjà été effectuées sur ces quatre plantes (Diallo et al., 2001; Cheng et al, 2002; Devi et al., 2002; Devi et al., 2003; Ojewole, 2003; Parimaladevi et al., 2003; Ojewole, 2004; Keita, 2005; Maïga et al., 2005; Adiza, 2007; Bose et al., 2011; Shah et al., 2011).

L'objectif de la présente étude était d'évaluer l'activité analgésique des extraits aqueux de Cymbopogon citratus (DC) Stapf, (Poaceae); Cleome viscosa Linn., (Capparaceae); Mitragyna inermis (Willd.) O. Kuntze (Rubiaceae) et Sclerocarya birrea (A. Rich.) Hochst (Anacardiaceae).

\section{MATERIEL ET METHODES}

\section{Matériel végétal}

Les rameaux feuillés de Mitragyna inermis (MIRF) et les écorces de tronc de
Sclerocarya birrea (SBET) ont été récoltés en octobre 2011 dans le cercle Kati, région de Koulikoro. Les feuilles de Cymbopogon citratus (CCF) et les parties aériennes de Cleome viscosa (CVPA) ont été aussi récoltées en octobre 2011 à Lomé.

Les échantillons des quatre plantes ont été identifiés au Département de Médecine Traditionnelle (DMT) de l'Institut National de Recherche en Santé Publique (INRSP) et des spécimens ont été déposés à l'herbier du DMT aux numéros 0077/DMT, 1548/DMT, 0033/DMT et 510/DMT respectivement pour Sclerocarya birrea, Cleome viscosa, Cymbopogon citratus et Mitragyna inermis. Les échantillons ont été séchés à l'ombre et à la température ambiante de la salle pendant dix jours. Chaque matériel végétal a ensuite été broyé à l'aide du broyeur Forplex type F1 disponible au DMT.

\section{Matériel animal}

Des souris femelles de type Oncins France Souche 1 (OF1), de poids compris entre 20 et $29 \mathrm{~g}$ ont été fournies par l'Animalerie du Centre National de Lutte contre la Maladie (CNAM). Les animaux ont été maintenus sous les conditions environnementales standard. Ils avaient un cycle de 12 heures de lumière/obscurité. Les animaux ont été nourris avec une alimentation pour souris préparée au niveau du DMT et ont eu un accès libre à l'eau de robinet.

Au total, nous avons utilisé soixante dix (70) souris reparties en quatorze lots de cinq souris dont trois lots pour les extraits de chaque plante, un lot témoin et un lot de référence. 


\section{Préparations des extraits}

Nous avons travaillé sur des extraits aqueux secs et des extraits aqueux extemporanés à $10 \%$ faits selon la forme d'utilisation en médecine traditionnelle.

\section{Extraits aqueux secs}

Décocté $10 \%$

Cent grammes de poudre de chaque plante ont été introduits dans $1000 \mathrm{ml}$ d'eau distillée puis l'ensemble a été porté à ébullition pour une durée de $15 \mathrm{mn}$. Le décocté obtenu, après refroidissement, a été filtré sur une compresse.

\section{Infusé 10\%}

Cent grammes de poudre de plante ont été versés dans $1000 \mathrm{ml}$ d'eau bouillante, après $15 \mathrm{mn}$ l'infusé a été filtré sur une compresse.

\section{Macéré aqueux $10 \%$}

Cent grammes de poudre de plante ont été macérés dans $1000 \mathrm{ml}$ d'eau distillée dans un erlenmeyer soumis à une agitation magnétique pendant $24 \mathrm{~h}$ à la température ambiante. Le macéré obtenu a été ensuite filtré sur une compresse.

Les filtrats (décocté, infusé et macéré) ont été par la suite concentrés au rotavapor à $50{ }^{\circ} \mathrm{C}$ sous vide puis congelés et lyophilisés.

Les extraits aqueux secs (décoctés, infusés et macérés) ont été pesés pour calculer le rendement de l'extraction.

\section{Extraits aqueux extemporanés}

Ces extraits ont été préparés selon les indications des formes d'utilisation traditionnelle pour chacune des quatre plantes (solution extemporanée à 10\%).

\section{Analyse phytochimique}

Les constituants chimiques des extraits ont été caractérisés à l'aide de chromatographie sur couche mince (CCM). A cet effet, les extraits ont été dissous (dissolution de $10 \mathrm{mg}$ dans $1 \mathrm{ml}$ du mélange méthanol : eau (1:1)) puis $10 \mu \mathrm{l}$ de solution diluée de chaque extrait ont été déposés sur des Plaques de Silicagel ${ }_{60} \mathrm{~F}_{254}$ qui ont été migrées dans le système Butanol-Acide acétique-eau ou BAW (60:15:25). Ensuite, les plaques ont été séchées puis observées à l'UV (254 nm et $365 \mathrm{~nm}$ ) avant leur révélation avec $\mathrm{FeCl}_{3}, \mathrm{AlCl}_{3}$, Dragendorff et Anisaldéhyde. Le facteur de rétention ou rapport frontal (Rf) de chaque tache a été ensuite calculé.

\section{Mode opératoire}

Les extraits ont été déposés à l'aide de micropipettes sur des plaques qui ont été migrées dans une cuve de CCM contenant la phase mobile (éluant) jusqu'au front du solvant préalablement tracé. Les chromatogrammes obtenus ont été observés et révélés avec les différents révélateurs. Les différentes taches ont été marquées selon leur mode d'apparition, localisations et leurs Rfs ont été calculés comme $d / D$ où $d=$ distance parcourue par la tache ; $\mathrm{D}=$ front du solvant.

\section{Activité analgésique}

Elle a été évaluée selon la méthode de la douleur provoquée par l'acide acétique ou le test de torsion (Sanogo et al., 2006). Les différents lots de souris ont été mis à jeun de 16 heures avec accès libre à l'eau avant le début du test. Douze lots ont été traités avec les extraits aqueux de chacune des quatre plantes aux doses de 100, $200 \mathrm{mg} / \mathrm{kg}$ et $25 \mathrm{ml} / \mathrm{kg}$ de solution extemporanée) de Cymbopogon citratus, Cleome viscosa, Mitragyna inermis et Sclerocarya birrea.

Un lot témoin a reçu de l'eau distillée à $25 \mathrm{ml} / \mathrm{kg}$.

Un lot de référence a été traité avec du Paracétamol à la dose de $150 \mathrm{mg} / \mathrm{kg}$.

Les extraits ont été administrés par voie intra-gastrique à l'aide d'une sonde métallique.

Une heure après les traitements, la douleur a été provoquée par une injection intra péritonéale de la solution d'acide acétique à $0,6 \%$ dans l'eau distillée à la dose de $10 \mu \mathrm{l} / \mathrm{g}$ selon la méthode décrite par (Sanogo et al., 2006).

Immédiatement après, chaque souris est mise dans une cage transparente et observée 
afin de noter le début de l'apparition des torsions douloureuses provoquées par l'acide acétique et leur nombre pendant une durée de 25 minutes.

\section{Expression des résultats et analyse statistique}

Les résultats sont exprimés sous forme de moyenne (M) des torsions effectuées dans chaque lot traité plus ou moins la déviation standard (DS): $(\mathrm{M} \pm \mathrm{DS})$. Les résultats ont été analysés par le test de $t$-Student et la significativité a été exprimée en $\mathrm{P}<0,05$ significatif et $\mathrm{P}<0,01$ très significatif par rapport au groupe témoin.

L'activité analgésique a été exprimée en pourcentage d'inhibition de la douleur pour chaque groupe traité par rapport au lot témoin qui n'a reçu que de l'eau distillée selon la formule suivante:

$\%$ Inhibition $=[(\mathrm{M}$ groupe témoin $-\mathrm{M}$ groupe traité) / M groupe témoin] x 100

\section{RESULTATS}

\section{Rendements des extraits}

Les extractions ont donné des rendements de $21,0 \% ; 13,5 \% ; 17,5 \%$ et $17,2 \%$ respectivement pour le Décocté MIRF, le Décocté SBET, l'Infusé CCF et le Macéré CVPA

\section{Constituants chimiques}

Les constituants chimiques des extraits des quatre plantes sont reportés au niveau de la Figure 1. Les taches noirâtres apparues sur les plaques révélées avec $\mathrm{FeCl}_{3}$ pourraient être des tanins (chromatogramme 1). L'apparition des taches jaunâtres à l'UV (366 nm) sur les plaques révélées avec $\mathrm{AlCl}_{3}$ pourrait indiquer la présence de flavonoïdes (chromatogramme 2). La présence des taches orangées sur les plaques révélées avec Dragendorff indiquerait les alcaloïdes (chromatogramme 3). Les taches bleu-clairs, violettes et jaunes apparues sur les plaques révélées avec anisaldéhyde pourraient être respectivement des terpènes, stérols et saponosides (chromatogramme 4).
Le chromatogramme 1 a mis en évidence la présence des tanins dans tous les extraits analysés alors que le chromatogramme 2 a montré leur richesse en flavonoïdes. Seules Mitragyna inermis et de Sclerocarya birrea possédaient des alcaloïdes (Chromatogramme 3). Les stérols étaient présents dans les extraits de Mitragyna inermis, Sclerocarya birrea et Cleome viscosa alors que les terpènes étaient absents dans les extraits de Mitragyna inermis et dans Cymbopogon citratus (Chromatogramme 4). Par ailleurs les saponosides n'étaient absents que dans Sclerocarya birrea (Chromatogramme 4).

\section{Activité analgésique}

Les extraits aqueux des quatre plantes protègent plus ou moins contre la douleur (Tableau 1). Mitragyna inermis aux doses de 100 et $200 \mathrm{mg} / \mathrm{kg}$ et son décocté extemporané a inhibé la douleur respectivement avec des pourcentages d'inhibition de 66,05\%, 39,04\% et $7,91 \%$. Le décocté de Sclerocarya birrea aux doses de 100 et $200 \mathrm{mg} / \mathrm{kg}$ et la solution extemporanée, ont inhibé respectivement la douleur avec $36,74 \%, 55,81 \%$ et $35,81 \%$. L'infusé de Cymbopogon citratus aux doses de 100 et $200 \mathrm{mg} / \mathrm{kg}$ a donné respectivement $45,58 \%$ et $76,74 \%$ d'inhibition et il a été obtenu avec la solution extemporanée une inhibition de $69,77 \%$. Le macéré aqueux de Cleome viscosa aux doses de 100 et 200 $\mathrm{mg} / \mathrm{kg}$ et sa solution extemporanée ont également inhibé la douleur avec respectivement $46,05 \%, 15,35 \%$ et $30,23 \%$. Dans les mêmes conditions, il a été obtenu une inhibition de la douleur de 73,02\% avec le Paracétamol à la dose de $150 \mathrm{mg} / \mathrm{kg}$.

Les extraits des espèces testées sur la douleur chez les souris protègent très significativement avec un effet dose dépendante. L'infusé de Cymbopogon citratus à la dose de $200 \mathrm{mg} / \mathrm{kg}$ a donné la meilleure inhibition de la douleur. 

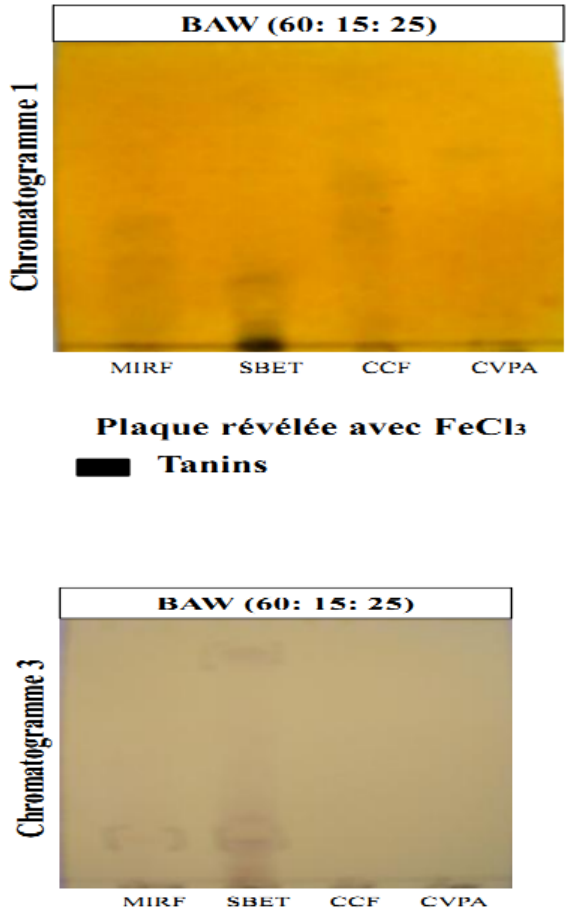

Plaque révélée avec Dragendorf

Alcalorides

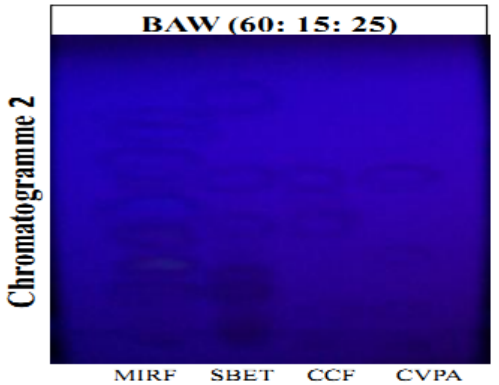

Plaque révélée avec $\mathrm{AlCl}_{3}$ et lue sous UV-366nm

Flavonoïdes

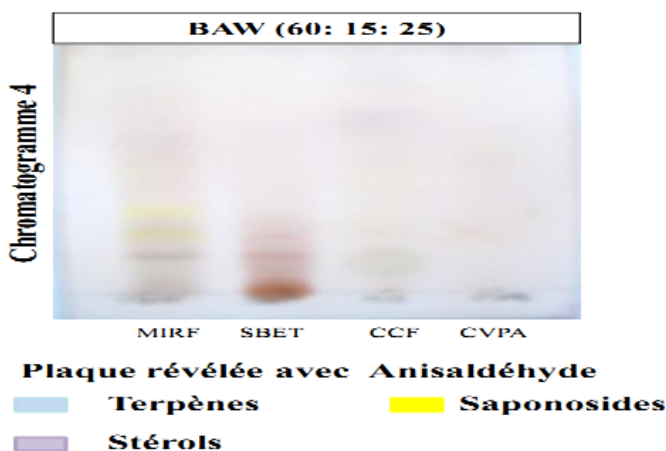

Figure 1: Récapitulatif de la phytochimie des quatre échantillons analysés.

Tableau 1: Activité analgésique des extraits de Cleome viscosa, Cymbopogon citratus, Mitragyna inermis et Sclerocarya bierra.

\begin{tabular}{lccc}
\hline Traitements & Doses mg/kg & $\begin{array}{c}\text { Nombre de torsions } \\
\text { M } \pm \mathbf{D S}\end{array}$ & \% Inhibition \\
\hline Eau distillée & $25 \mathrm{ml} / \mathrm{kg}$ & $43,00 \pm 2,55$ & - \\
& Solution extemporanée & $39,60 \pm 0,55$ & 7,91 \\
Décocté MIRF & 100 & $14,60 \pm 3,29 * *$ & 66,05 \\
& 200 & $26,20 \pm 4,55^{*}$ & 39,07 \\
Décocté SBET & Solution extemporanée & $27,60 \pm 1,95^{*}$ & 35,81 \\
& 100 & $27,20 \pm 3,63^{*}$ & 36,74 \\
Infusé CCF & 200 & $19,00 \pm 6,96^{*}$ & 55,81 \\
& Solution extemporanée & $13,00 \pm 4,90^{* *}$ & 69,77 \\
Macéré aqueux & 100 & $23,40 \pm 6,99 *$ & 45,58 \\
CVPA & 200 & $10,00 \pm 8,80 * *$ & 76,74 \\
Paracétamol & Solution extemporanée & $30,00 \pm 6,48^{*}$ & 30,23 \\
\hline M: moyenne, DS: déviation standard, **p<0,01:très significatif, *p<0,05: significatif, p $>0,05:$ non significatif.
\end{tabular}




\section{DISCUSSION}

La douleur palustre est l'un des signes qui amènent le patient en consultation et elle nécessite une prise en charge conventionnelle ou traditionnelle.

Dans les conditions du présent travail, les rameaux feuillés de Mitragyna inermis ont donné le meilleur rendement d'extraction avec l'eau. L'analyse phytochimique a révélé que les quatre plantes sont riches en tanins, flavonoïdes, alcaloïdes, stérols et terpènes, des saponosides et en substances antiradicalaires. Ces constituants hydrosolubles sont certainement les substances actives. Avec les feuilles de Mitragyna inermis, Konkon et al. (2008) ont signalé que les polyphénols, les flavonoïdes et tanins catechiques sont présents dans les extraits méthanolique, infusé et décocté mais absents dans l'extrait chloroformique. Les saponosides sont présents seulement dans le décocté de la plante tandis que les stérols, triterpènes et alcaloïdes étaient dans tous les extraits. Adiza (2007), par la chromatographie sur couche mince a révélé la présence de tanins et flavonoïdes dans l'infusé des écorces de tronc de Sclerocarya birrea (A. Rich.) Hochst. Les extraits aqueux et éthanolique des feuilles de Cymbopogon citratus (DC) Stapf, sont riches en tanins, flavonoïdes, hydrates de carbone, glycosides cardiaques, stérols, anthraquinones et alcaloïdes (Sha'a et al., 2011). Certains auteurs ont signalé la présence des alcaloïdes, des tanins, des flavonoïdes, des saponosides et des terpènes dans les feuilles de Cleome viscosa (Koche et al., 2010).

La plus forte activité analgésique a été obtenue avec les extraits aqueux secs et la solution extemporanée de Cymbopogon citratus. Cependant, l'extrait aqueux sec de Mitrgyna inermis à la dose de $100 \mathrm{mg} / \mathrm{kg}$ a donné une inhibition de $66,05 \%$ alors que l'extrait aqueux sec de Cleome viscosa (200 $\mathrm{mg} / \mathrm{kg}$ ) n'avait pas une importante activité.

Sha'a et al. (2011) ont trouvé que 100 $\mathrm{mg} / \mathrm{kg}$ des extraits aqueux et éthanolique de Cymbopogon citratus, inhibaient la douleur respectivement avec $54,1 \%$ et $55,4 \%$. Par ailleurs, l'infusé, les flavonoïdes et les tanins de cette plante avaient respectivement réduit la douleur avec $57,0 \%, 54,60 \%$ et $52,20 \%$ (Garcia et al., 2015). En Afrique du sud, Ojewole (2004) a trouvé que les extraits aqueux des écorces de tronc Sclerocarya birrea administrés par voie orale à des doses allant de 100 à $800 \mathrm{mg} / \mathrm{kg}$ présentaient une protection dose dépendante contre la douleur provoquée par la chaleur. L'extrait méthanolique de Cleome viscosa a montré un pouvoir analgésique (Parimaladevi et al., 2003). L'activité analgésique de l'extrait méthanolique des feuilles de Cleome viscosa, étudiée par Bose et al. (2011), a donné à la dose de $500 \mathrm{mg} / \mathrm{kg}$ une inhibition de 47,94\%. Ces résultats concordent avec ceux des études antérieures sauf Mitragyna inermis qui à notre connaissance n'a pas été étudiée pour ces propriétés analgésiques en Afrique. Cependant, la solution extemporanée de Mitragyna inermis a donné une importante activité tandis que celle du macéré aqueux de Cleome viscosa à la dose de $200 \mathrm{mg} / \mathrm{kg}$ est non importante. Les extraits aqueux des plantes testées agissent de façon dose dépendante et cela confirme les résultats des travaux de Boye et al. (2014) sur les extraits aqueux de Corchorus olitorius et ceux de Kouakou et al. (2010) sur Mitracarpus scaber. Par ailleurs, certains travaux avaient montré l'activité analgésique d'extraits de saponosides (Chindo et al., 2010; Adiukwu et al., 2012). L'activité analgésique de Cymbopogon citratus du présent travail est similaire à celui de Kouakou et al. (2010) qui ont montré que $10 \mathrm{mg} / \mathrm{kg}$ d'extrait aqueux de Mitracarpus scaber avaient un effet analgésique supérieur à celui du kétoprofène.

\section{Conclusion}

Dans l'ensemble, l'activité analgésique évaluée avec Cymbopogon citratus, Cleome viscosa, Mitragyna inermis et Sclerocarya birrea a montré des effets très significatifs sur la réduction de la douleur provoquée chez les souris. Cymbopogon citratus et Mitragyna inermis ont présenté les meilleures activités. Les groupes chimiques mis en évidence dans les différents extraits des plantes testées 
pourront être à l'origine des activités constatées. Les résultats de cette étude constituent donc une base solide pour la valorisation des médecines traditionnelles malienne et togolaise dans la lutte efficace et durable de la douleur palustre.

Les investigations phytochimiques des plantes testées seront poursuivies par des fractionnements en vue de l'identification des molécules de chaque groupe chimique présent responsable des activités analgésiques. Des tests toxicologiques seront aussi envisager pour évaluer l'innocuité de ces plantes dans le souci d'aboutir à la production de médicaments traditionnels améliorés.

\section{CONFLIT D'INTERETS}

Les auteurs déclarent une absence de conflit d'intérêts.

\section{CONTRIBUTIONS DES AUTEURS}

$\mathrm{AD}$ a participé à la récolte du matériel végétal, la préparation des extraits, l'expérimentation, la saisie des données et la rédaction du manuscrit. KK a participé à la récolte du matériel végétal, l'analyse des données et la correction du manuscrit. MH a participé à l'expérimentation. AT a corrigé le manuscrit. RS a participé à l'expérimentation, l'analyse des données et la correction du manuscrit. KE a participé à la correction du manuscrit. KAA a aussi corrigé le manuscrit. DD a corrigé le manuscrit final. MG a participé à la correction du manuscrit.

\section{REMERCIEMENTS}

Les auteurs remercient les techniciens du département médecine traditionnelle pour les efforts consentis.

\section{REFERENCES}

Adiukwu PC, Amon A, Nambatya G, Adzu B, Imanirampa L, Twinomujuni $\mathrm{S}$, Twikirize O, Amanya M, Ezeonwumelu OJ, Oloro J, Okoruwa GA, Katusiime B. 2012. Acute toxicity, antipyretic and antinociceptive study of the crude saponin from an edible vegetable: Vernonia amygdalina leaf.
Int. J. Biol. Chem. Sci., 6(3): 10191028.

Adiza A. 2007. Etude d'une recette traditionnelle, des écorces de tronc de Sclerocarya birrea Hosch et de Uapaca togoensis Pax utilisées dans le traitement du diabète. Thèse de Pharmacie, FMPOS, Bamako, 107p.

Aubry P. 2013. Paludisme, actualités 2013. Diplôme de médecine tropicale des pays de l'Océan Indian. Médecine Tropicale. 22p.

Bose U, Bala V, Ghosh TN, Gunasekaran K, Ahmed Ayedur Rahman AA. 2011. Antinociceptive, cytotoxic and antibacterial activities of Cleome viscosa leaves. Rev. Bras. Farmacogn. Braz. J. Pharmacogn., 21(1): 165-169.

Boye A, Barku VYA, Amoateng P Mbroh LA. 2014. Anti-nociceptive and antioxidant activities of an aqueous leaf extract of Corchorus olitorius L. (Tiliaceae). Int. J. Biol. Chem. Sci., 8(6): 2395-2406.

Chindo BA, Joseph A. Anuka JA, Isaac E, Ahmadu AA, Tarfa FD, Gamaniel SK. 2010. Saponins are involved in the analgesic and anti-inflammatory properties of Ficus platyphylla stem bark. Int. J. Biol. Chem. Sci., 4(2): 415423.

Cheng ZH, Yu BH, Yang XW. 2002. 27-Nortriterpenoid glucosides from Mitragyna Inermis. Phytochemistry, 61: 379-382.

Devi BP, Boominathan R, Mandal SC. 2002. Evaluation of anti-diarrheal activity of Cleome viscosa L. extract in rats. Phytomedicine, 9: 739-742.

Devi BP, Boominathan R, Subhash Mandal C. 2003. Studies of pharmacognostical profiles of Cleome viscosa $L$. (Capparidaceae). Ancient Science of Life, 22(4): 115-121.

Diallo D, Marston A, Terreaux C, Touré Y, Paulsen BS, Hostettmann K. 2001. Screening of Malian medicinal plants for antifungal larvicidal, molluscicidal, antioxidant and Radical Scavenging 
activities. Phytotherapy Research., 15 : 401- 406.

Garcia R, Ferreira J, Costa G, Santos T, Branco FS, Caramona MM. 2015. Evaluation of anti- inflammatory and analgesic activities of Cymbopogon citratus in vivo polyphenols contribution. Research Journal of Medicinal Plant, 9(1): 1-13.

Keita A. 2005. Etude de trois plantes utilisées dans le traitement traditionnel de l'ulcère gastro-duodénal dans le district de Bamako: Borassus oethiopum Mart (Palmeae), Sclerocarya birrea (A. Rich.) Hochst. (Anacardiaceae) et Ximenia americana L. (Olacaceae), Thèse de pharmacie, FMPOS, Bamako, 173p.

Koche D, shirsat R, Imran S, Bhadange DG. 2010. Phytochemical screening of eight traditionally used Ethnomedicinal plants from Akola District (MS) India. International Journal of Pharma and Bio Sciences, 1(4): 253-256.

Konkon NG, Adjoungoua AL, Manda P, Simaga D, N'Guessan K, Kone BD. 2008. Toxicological and phytochemical screening study of Mitragyna Inermis (willd.) O.ktze (Rubiaceae), antidiabetic plant. Journal of Medicinal Plants Research, 2(10): 279-284.

Kouakou SL, Kouakou GS, Laba ID, Brou JK. 2010. Evaluation de l'activité analgésique de l'extrait aqueux des feuilles de Mitracarpus scaber Zucc (Rubiacées), une plante médicinale de Côte d'Ivoire. Int. J. Biol. Chem. Sci., 4(2): 456-463.

Maïga A, Diallo D, Fané S, Sanogo R, Paulsen BS, Cissé B, 2005. A survey of toxic plants on the market in the district of Bamako, Mali: traditional knowledge compared with a literature search of modern pharmacology and toxicology. Journal of Ethnopharmacology, 96: 183-193.
Okunji CO, Acton N, Ellis WY, Iwu MM. 2000. Identification of new antimalarial pharmacophores from West and Central African plants. Proceedings of the International Conference on Traditional Medicine for HIV/AIDS and Malaria. Nicon Hilton Hotel, Abuja Nigeria.

Ojewole JA. 2003. Evaluation of the antiinflammatory properties of Sclerocarya birrea (A. Rich) Hochst. (Family: Anacardiaceae) stem-bark extracts in rats. Journal Ethnopharmacology, 85: 217- 220.

Ojewole JA. 2004. Evaluation of the analgesic, anti-inflammatory and antidiabetic properties of Sclerocarya birrea (A. Rich) Hochst. Stem-bark aqueous extract in mice and rats. Phytother. Res., 18(8): 601-608.

Parimaladevi B, Boominathan R, Mandal SC, 2003. Studies on analgesic activity of Cleome viscosa in mice. Fitoterapia, 74(3): 262-266.

Sanogo R, Maïga A, Diallo D. 2006. Activités analgésique et anti-ininflammatoire des extraits de Maytenus senegalensis, Stereospermum kuntrianum et Tricrilia emetica utilisées dans le traitement traditionnel des dysmenorrhees au Mali. Pharm. Méd. Trad. Afr., 14: 123136.

Shah G, Shri R, Panchal V, Sharma N, Singh B, Mann AS. 2011. Scientific basis for the therapeutic use of Cymbopogon citratus, Stapf (Lemon grass). Review Article, 2: 3-8.

WHO. 2013. World Malaria Report. World Health Organization: Geneva. 